\title{
Liquid chromatography mass spectrometry- based profiling of phosphatidylcholine and phosphatidylethanolamine in the plasma and liver of acetaminophen-induced liver injured mice
}

Ya-Nan Ming ${ }^{1 \dagger}$, Jing-Yi Zhang ${ }^{1 \dagger}$, Xiao-Lin Wang ${ }^{2}$, Chun-Min Li ${ }^{1}$, Si-Cong Ma ${ }^{3}$, Zheng-Yang Wang ${ }^{4}$, Xiao-Lin Liu ${ }^{5}$, Xiao-Bo Li ${ }^{4^{*}}$ and Yi-Min Mao ${ }^{1 *}$ (D)

\begin{abstract}
Background: Acetaminophen (APAP) overdose is one of the most common causes of acute liver failure in many countries. The aim of the study was to describe the profiling of phosphatidylcholine (PC) and phosphatidylethanolamine (PE) in the plasma and liver of Acetaminophen -induced liver injured mice.

Methods: A time course study was carried out using C57BL/6 mice after intraperitoneal administration of 300 mg/kg Acetaminophen 1 h, 3 h, 6 h, 12 h and 24 h. A high-throughput liquid chromatography mass spectrometry (LC-MS) lipidomic method was utilized to detect phosphatidylcholine and phosphatidylethanolamine species in the plasma and liver. The expressions of phosphatidylcholine and phosphatidylethanolamine metabolism related genes in liver were detected by quantitative Reverse transcription polymerase chain reaction (GRT-PCR) and Western-blot.

Results: Following Acetaminophen treatment, the content of many PC and PE species in plasma increased from $1 \mathrm{~h}$ time point, peaked at $3 \mathrm{~h}$ or $6 \mathrm{~h}$, and tended to return to baseline at $24 \mathrm{~h}$ time point. The relative contents of almost all PC species in liver decreased from $1 \mathrm{~h}$, appeared to be lowest at $6 \mathrm{~h}$, and then return to normality at $24 \mathrm{~h}$, which might be partly explained by the suppression of phospholipases mRNA expressions and the induction of choline kinase (Chka) expression. Inconsistent with PC profile, the relative contents of many PE species in liver increased upon Acetaminophen treatment, which might be caused by the down-regulation of phosphatidylethanolamine N-methyltransferase (Pemt).
\end{abstract}

Conclusions: Acetaminophen overdose induced dramatic change of many PC and PE species in plasma and liver, which might be caused by damaging hepatocytes and interfering the phospholipid metabolism in Acetaminophen -injured liver.

Keywords: Acetaminophen, Phosphatidylcholine, Phosphatidylethanolamine, Lipidomics, Liver injury

\footnotetext{
*Correspondence: xbli@fudan.edu.cn; maoym11968@163.com

${ }^{\dagger}$ Equal contributors

${ }^{4}$ Department of Physiology and Pathophysiology, School of Basic Medical

Sciences, Fudan University, Shanghai 200032, China

'Division of Gastroenterology and Hepatology, Renji Hospital, School of

Medicine, Shanghai Jiao Tong University, Shanghai Institute of Digestive

Disease, Shanghai, China

Full list of author information is available at the end of the article
} 


\section{Background}

As a major site for drug metabolism and elimination, the liver is susceptible to drug toxicity. Drug-induced liver injury (DILI) is a significant clinical problem and a challenge for drug development worldwide. Acetaminophen (N-acetyl-p-aminophenol, APAP) is commonly used as an over-the-counter analgesic and antipyretic drug known to be safe at therapeutic doses. However, APAP overdose has become one of the most common causes of acute liver failure in many countries [1]. APAPinduced liver injury is the most frequent drug hepatotoxicity and the most used experimental model of DILI. The mechanism of APAP-induced liver injury is complicated and not fully understood. The accumulation of $\mathrm{N}$ acetyl-p-benzoquinone imine (NAPQI), the reactive and toxic metabolite of APAP, is considered as the main cause of liver injury induced by overdose of APAP. In mouse APAP-induced models and in human, the reaction of NAPQI with protein sulfhydryl groups of cysteine might trigger mitochondrial damage, oxidative stress, cjun N-terminal kinase (JNK) activation, the nuclear DNA fragmentation and cell death [2-4].

In mammalian cells, phosphatidylcholine (PC) and phosphatidylethanolamine (PE) are the first and second most abundant phospholipid respectively. In liver, PC is the principal component of cellular membrane, a precursor of signaling molecules, and a key element of lipoproteins and bile. In addition to its structural role in membrane and as a substrate for methylation to $\mathrm{PC}$ in the liver, $\mathrm{PE}$ is also a substrate for anandamide synthesis, regulates membrane fusion, and supplies ethanolamine for glycosylphosphatidylinositol anchors of cell-surface signaling proteins. Previous studies over 50 years ago reported the contents of phosphatidylcholine (PC) and phosphatidylethanolamine (PE) were decreased,but the $\mathrm{PE}: \mathrm{PC}$ ratio was elevated in the liver of rats challenged with $\mathrm{CCl}_{4}[5,6]$. Lipidomics is a powerful technology defined as the complete quantitative and molecular determination of lipid molecules isolated from cells, tissue or biological fluid [7-9]. During APAP-induced liver injury, lipidome is the overall results of cellular/ subcellular dysfunction and alterations of larges molecules like proteins/enzymes/DNA, and consequently more easily correlated with the phenotype. The analysis of lipidome by lipidomics direct to a more detailed understanding of biochemical changes during DILI.

Recent years, emerging lipidomic techniques were used to describe comprehensive and global PC/PE profiles in plasma/liver of experimental animals with drug/chemicalinduced liver injury. Several studies reported that serum levels of certain PCs/PEs were significantly changed in acute rat liver injury induced by chemicals such as APAP, CCL4, galactosamine and ricinine [10-12]. Cheng J et al. analyzed APAP-treated mice serum through LC-MSbased metabolomics, and they found that C20:4-LPC gradually declined with rising liver toxicity [13]. Xie $\mathrm{T}$ et al. observed the remodeling of $\mathrm{PC} / \mathrm{PE}$ in liver of rats with Tripterygium wilfordii induced liver injury. The alternations comprised fatty acid composition of PC changed and increased Lyso PC and certain PE decreased, which might affect membrane fluidity, the inflammatory reaction, and mitochondria dysfunction respectively [14]. However, the alternation of individual PC/PE specie in both plasma and liver of APAP-injured mice and correlations of PCs/PEs between plasma and liver remains unknown. We already developed an LC-MS based lipidomic method for simultaneous detection of diverse lipids [15]. In the present study, using this high-throughput method, we compared the relative concentrations of phosphatidylcholine and phosphatidylethanolamine in the plasma and liver of the APAP-induced liver injured mice and salinetreated control mice at series of time-points, and then measured the expressions of genes involved in $\mathrm{PC} / \mathrm{PE}$ metabolism in liver. The experimental work is focusing on the search for possible mechanisms leading to hepatotoxicity than on biomarkers indicating an APAP intoxication.

\section{Results}

\section{Biochemical assays and H\&E staining}

Plasma levels of ALT and AST were measured to evaluate APAP-induced acute liver injury. As shown in Fig.1a and b, plasma ALT and AST levels increased in $300 \mathrm{mg} / \mathrm{kg}$ APAP induced acute liver injury mice model at all five time points $(1 \mathrm{~h}, 3 \mathrm{~h}, 6 \mathrm{~h}, 12 \mathrm{~h}$ and $24 \mathrm{~h})$. The most significant increase of ALT/AST was at $6 \mathrm{~h}$ time point. In histological evaluation, as shown in Fig.1c, APAP-treated mice displayed centrilobular hepatic necrosis, hyperemia of the hepatic sinus, presence of inflammatory infiltrate, pyknotic nucleus, cytoplasm vacuolization and loss of cell boundaries whereas saline-treated mice at any time point showed normal liver histology. The prominent morphological damage started at $3 \mathrm{~h}$, deteriorated with time, and then attenuated at $24 \mathrm{~h}$ time point (Fig.1d).

\section{Phosphatidylcholine and phosphatidylethanolamine profiling in mice plasma analyzed by LC-MS}

The relative concentrations of $57 \mathrm{PC} / \mathrm{LPC}$ species and $18 \mathrm{PE} / \mathrm{LPE}$ species in plasma were simultaneously determined using LC-MS method. The typical positive total ion chromatograms (TIC) of mouse liver were shown in Fig. 2. The original data of $\mathrm{PC} / \mathrm{PE}$ concentrations in plasma were shown in Supplemental Additional file 1: Table S1. The 75 phospholipids were loaded into a PCA model. As shown in Fig. 3a and c, the APAP-treated group could be clearly distinguished from the salinetreated group at $3 \mathrm{~h}$ time point as well as at $6 \mathrm{~h}$ time 


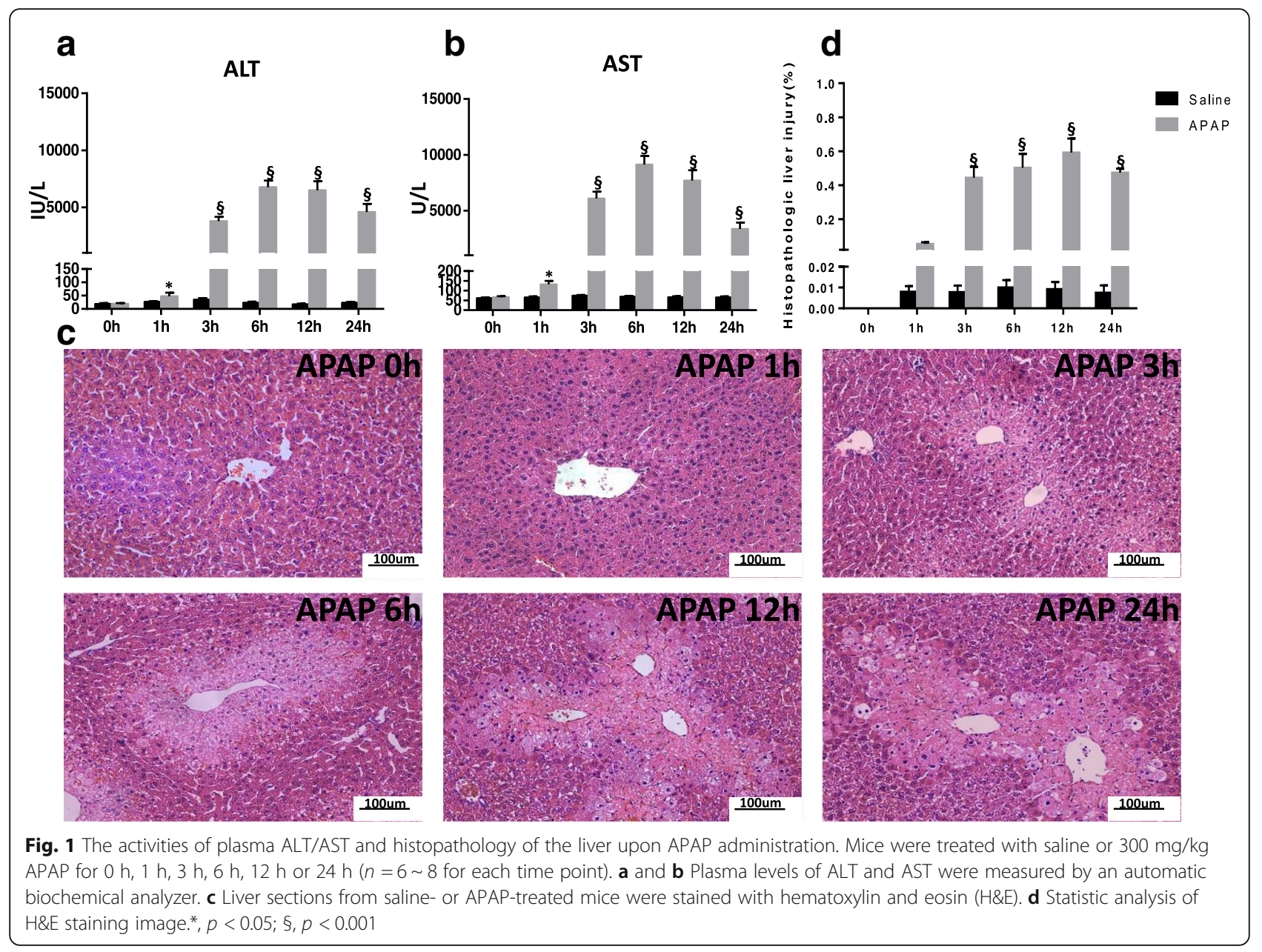

point according to the PCA score plots. These variables were further loaded into a PLS-DA model. As shown in Fig. $3 \mathrm{~b}$ and d, the differences between saline- and APAPtreated samples were also depicted by PLS-DA score plot at $3 \mathrm{~h}$ time point or $6 \mathrm{~h}$ time point. The above score plots suggested that the metabolic pattern of $\mathrm{PC} / \mathrm{PE}$ species in plasma was altered by APAP treatment at both $3 \mathrm{~h}$ and $6 \mathrm{~h}$.

The ratio of lipid concentrations in APAP-treated group/lipid concentrations in saline-treated group at

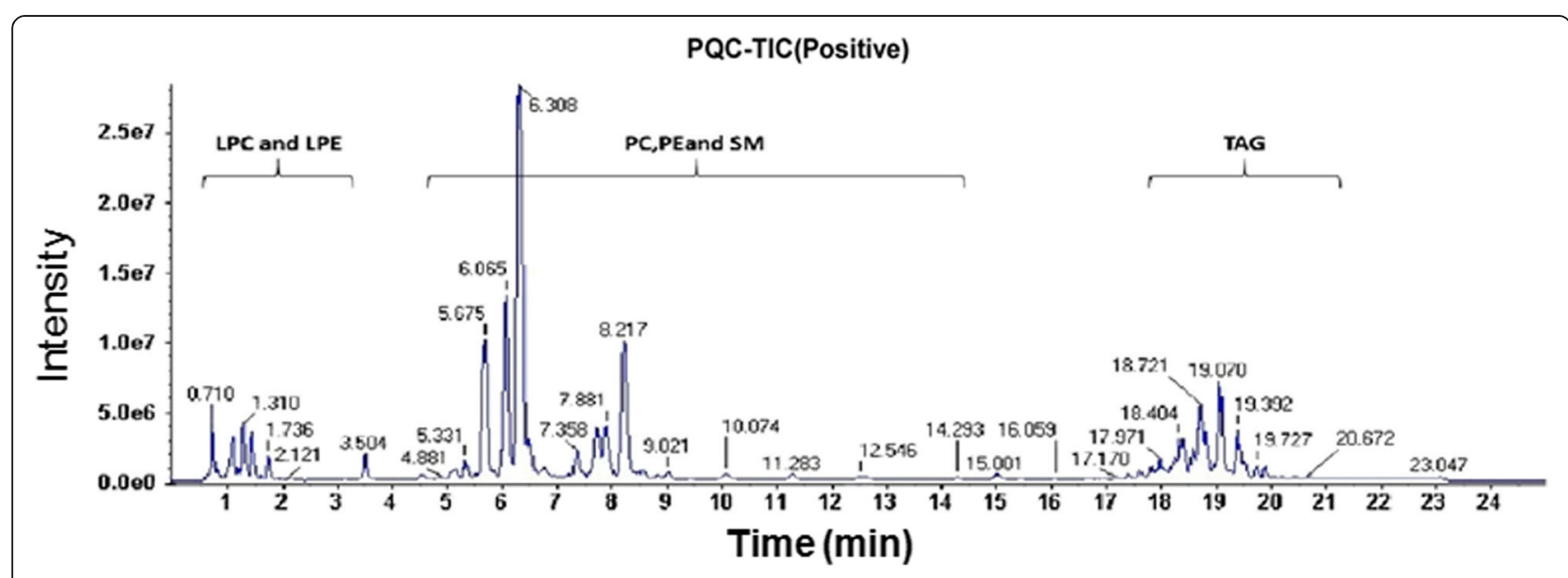

Fig. 2 Total ions chromatogram (TIC) of mouse plasma sample 

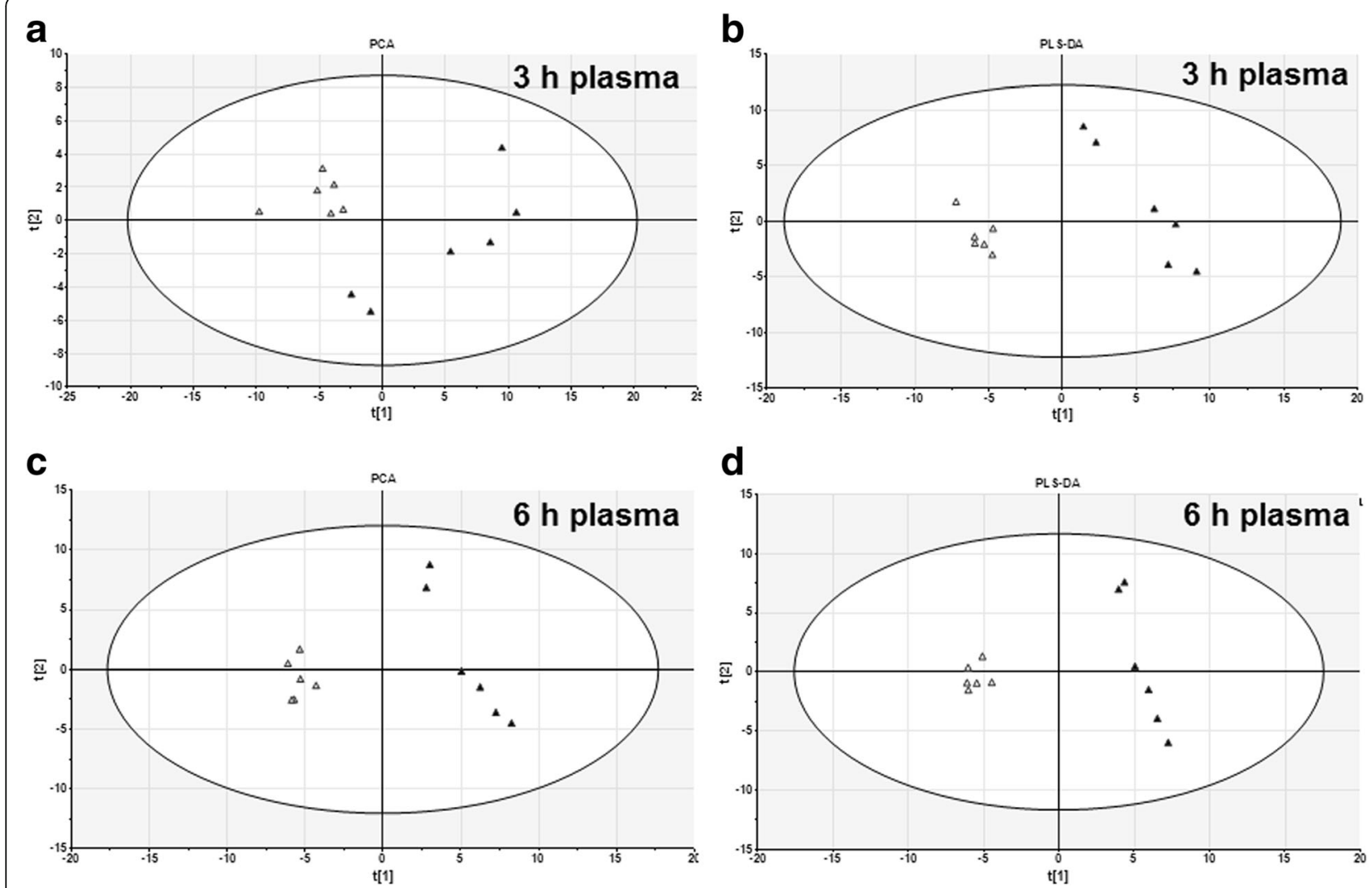

Fig. 3 PCA and PLS-DA score plot based on the plasma PC and PE profiling of APAP-induced liver injured mice. Seventy-five variables (57 PC/LPC and 18 PE/LPE) at $3 \mathrm{~h}(\mathbf{a}, \mathbf{c})$ and $6 \mathrm{~h}(\mathbf{b}, \mathbf{d})$ time points were loaded into a PCA $(\mathbf{a}, \mathbf{b})$ model and a PLS-DA (c, d) model. The score plot indicated a clear separation between the saline- $(\triangle)$ and APAP-treated $(\boldsymbol{\Lambda})$ groups

each time-point was calculated and represented as the relative concentrations of APAP-treated group in Additional file 1: Table S1. The fold-changes of PC/PE species in mouse plasma upon APAP treatment were also illustrated by heat map in Supplemental Additional file 2: Figure S1A (PC) and Figure S1B (PE).

Figure 4a summarized the numbers of decreased and increased PCs/LPCs/PEs/LPEs in plasma upon APAPtreatment. We defined lipid species with statistically significant increase or decrease at least at one time-point among $1 \mathrm{~h}, 3 \mathrm{~h}$ and $6 \mathrm{~h}$ as the increased or decreased lipid species respectively. The increased phospholipids are much more than decreased phospholipids in plasma upon APAP treatment. As shown in Fig. 4b and c, PC 33:1, PC 34:3, PE 34:2, PE 36:3, PE 38:4 and PE 38:6 were elevated significantly both at $3 \mathrm{~h}$ and $6 \mathrm{~h}$ time points upon APAP treatment. Pearson's correlation was performed to analyze the correlation between these 6 phospholipid species and liver enzymes (ALT/ AST) which were normally used to be elevated in DILI. As showed in Fig. 4d, the increases of these 6 phospholipids are positively correlated with the changes of ALT/AST.

\section{The profiles of PC and PE in mice livers analyzed by LC-MS}

To investigate the mechanism of $\mathrm{PC} / \mathrm{PE}$ changing in plasma, we detected the $\mathrm{PC} / \mathrm{PE}$ profiles in livers of APAP- and saline-treated mice by LC-MS. The levels of $63 \mathrm{PC} / \mathrm{LPC}$ species and $43 \mathrm{PE} / \mathrm{LPE}$ species in liver were simultaneously determined using LC-MS method. The analytical data's of $106 \mathrm{PC} / \mathrm{PE}$ species were loaded into a PCA and a PLS-DA model. As shown in Fig. 5a/c (PCA model) and Fig. 5b/d (PLS-DA model), the differences between control group and APAP group were nearly identical in both data models. The above score plots suggested that the metabolic pattern of PC and PE in livers was altered by APAP treatment at both $3 \mathrm{~h}$ and $6 \mathrm{~h}$.

The fold-changes of $\mathrm{PC} / \mathrm{PE}$ species in mouse liver upon APAP treatment were also illustrated by heat map in Additional file 3: Figure S2A (PC) and figure S2B $(\mathrm{PE})$. Figure $6 \mathrm{a}$ summarized the numbers of decreased and increased PCs/LPCs/PEs/LPEs in liver upon APAPtreatment. We defined lipid species with statistically significant decrease or increase at least at one time-point among $1 \mathrm{~h}, 3 \mathrm{~h}$ and $6 \mathrm{~h}$ as the decreased or increased lipid species respectively. The decreased phospholipids 


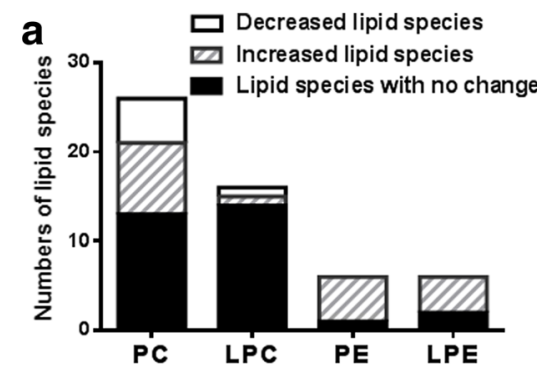

C

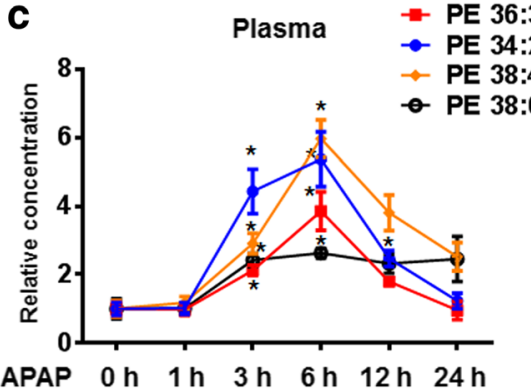

b

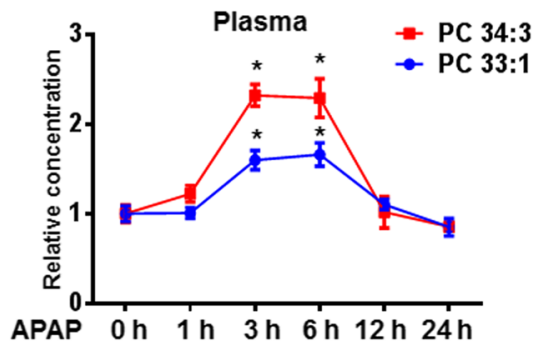

d

\begin{tabular}{|l|c|r|r|r|}
\hline & \multicolumn{2}{|c|}{ ALT } & \multicolumn{2}{c|}{ AST } \\
\hline & $\begin{array}{c}\text { Pearson } \\
\text { correaltion } \\
\text { coefficient }\end{array}$ & P value & $\begin{array}{c}\text { Pearson } \\
\text { correaltion } \\
\text { coefficient }\end{array}$ & P value \\
\hline PC 33:1 & $0.414^{*}$ & 0.017 & $0.566^{* *}$ & 0.001 \\
\hline PC 34:3 & 0.322 & 0.064 & $0.484^{* *}$ & 0.004 \\
\hline PE 34:2 & $0.592^{* *}$ & $\mathrm{P}<0.01$ & $0.709 * *$ & $\mathrm{P}<0.01$ \\
\hline PE 36:3 & $0.613^{* *}$ & $\mathrm{P}<0.01$ & $0.690^{* *}$ & $\mathrm{P}<0.01$ \\
\hline PE 38:4 & $0.723^{* *}$ & $\mathrm{P}<0.01$ & $0.802^{* *}$ & $\mathrm{P}<0.01$ \\
\hline PE 38:6 & $0.697^{* *}$ & $\mathrm{P}<0.01$ & $0.836^{* *}$ & $\mathrm{P}<0.01$ \\
\hline
\end{tabular}

Fig. 4 PC and PE profiles in the plasma of APAP-treated mice. a The numbers of decreased and increased PCs/LPCs/PEs/LPEs in plasma upon APAP-treatment were summarized. We defined lipid species with statistically significant decrease or increase at least at one time-point among 1 h, 3 h and $6 \mathrm{~h}$ as the decreased or increased lipid species respectively. $\mathbf{b}$ and $\mathbf{c} 2$ PCs (PC 33:1 and PC 34:3) and 4 PES (PE 34:2, PE 36:3, PE 38:4 and PE 38:6) were increased significantly in APAP-treated group compared with saline-treated group at both $3 \mathrm{~h}$ and $6 \mathrm{~h}$ time points. The relative concentrations of these 6 phospholipids in plasma of APAP induced liver-injured mice at 0 h, 1 h, 3 h, 6 h, 12 h and 24 h time points were presented. $\mathbf{d}$ Pearson's correlation was performed to analyze the correlation between these 6 phospholipid species and liver enzymes (ALT/AST). * statistically significant compared with saline-treated group

are much more than increased phospholipids in liver upon APAP treatment, which are opposite to profiles in plasma. We picked out the decreased PCs/LPCs/PEs/ LPEs in liver, and then performed Scatter Plot of these phospholipids both in liver and plasma. As shown in Fig. 6b, most of decreased PCs and LPEs in liver might cause their increases in plasma. Because the significantly changed PEs in liver couldn't be detected in plasma, PEs were not shown in Fig. 6b. Furthermore, the relative concentrations of the 6 increased plasma phospholipids in liver were plotted in Fig. 6c and d. PC 33:1 and PC 34:3 in liver decreased significantly. PE 34:2 and PE 36:3 in liver slightly decreased, while PE 38:4 and PE 38:6 in liver slightly increased.

\section{The expression pattern of PC and PE metabolism related} genes in APAP-injured mice livers

To further investigate the mechanism of PC/PE changing in liver, we measured the expression of genes involved in PC/PE metabolism in liver tissue. There are over 40 different phospholipases in liver. Based on our previous RNAseq data, 13 phospholipases including 1 PLA1 (Pla1a), 8 PLA2 (Pla2g6, Pla2g7, Pla2g12a, Pla2g12b, Pla2g15, Pnpla2, Pnpla7, Pnpla8), 2 PLC (Plcg1, Plcxd2) and 2 PLD (Pld3, Pld4) are relatively abundant in mouse liver. The mRNA levels of these 13 phospholipases in livers of saline- or APAP-treated mice were detected by
qRT-PCR. As shown in Fig. 7a, the concentration of mRNA of most phospholipases in liver decreased upon APAP treatment for $3 \mathrm{~h}$ or $6 \mathrm{~h}$. We also measured the mRNA levels of PC/PE synthesis related genes. Among these genes, Pemt was decreased, while Chka was uniquely increased in APAP-injured livers (Fig. 7b). As shown in Fig. 7c, the induction of Chka was $~ 3.5$-fold at $1 \mathrm{~h}$, peaked at $6 \mathrm{~h}(\sim 16$-fold $)$ and then went back to baseline at $24 \mathrm{~h}$ in APAP-injured livers. Western blot assays of the liver homogenates demonstrated Chka in livers upon APAP treatement was increased timedependently, appeared to be greatest at the $12 \mathrm{~h}$ time point, which is about several hours-delayed compared with Chka mRNA induction (Fig. 7d).

\section{Discussion}

In the present study, we utilized the high-throughput LC-MS lipidomic method to acquire PC and PE profiles both in plasma and liver of APAP-induced liver injured mice at different time points after dosing.

The mice model of APAP-induced liver injury mostly resembles the human pathophysiology of both liver injury and recovery [16]. In our study, the sub-lethal dose of APAP $(300 \mathrm{mg} / \mathrm{kg}$ ) was used to induce liver injury in mice. In this model, the most significant increase of ALT/AST is at $6 \mathrm{~h}$ time point. At $24 \mathrm{~h}$ time point, the liver function presented the recovery situation verified 

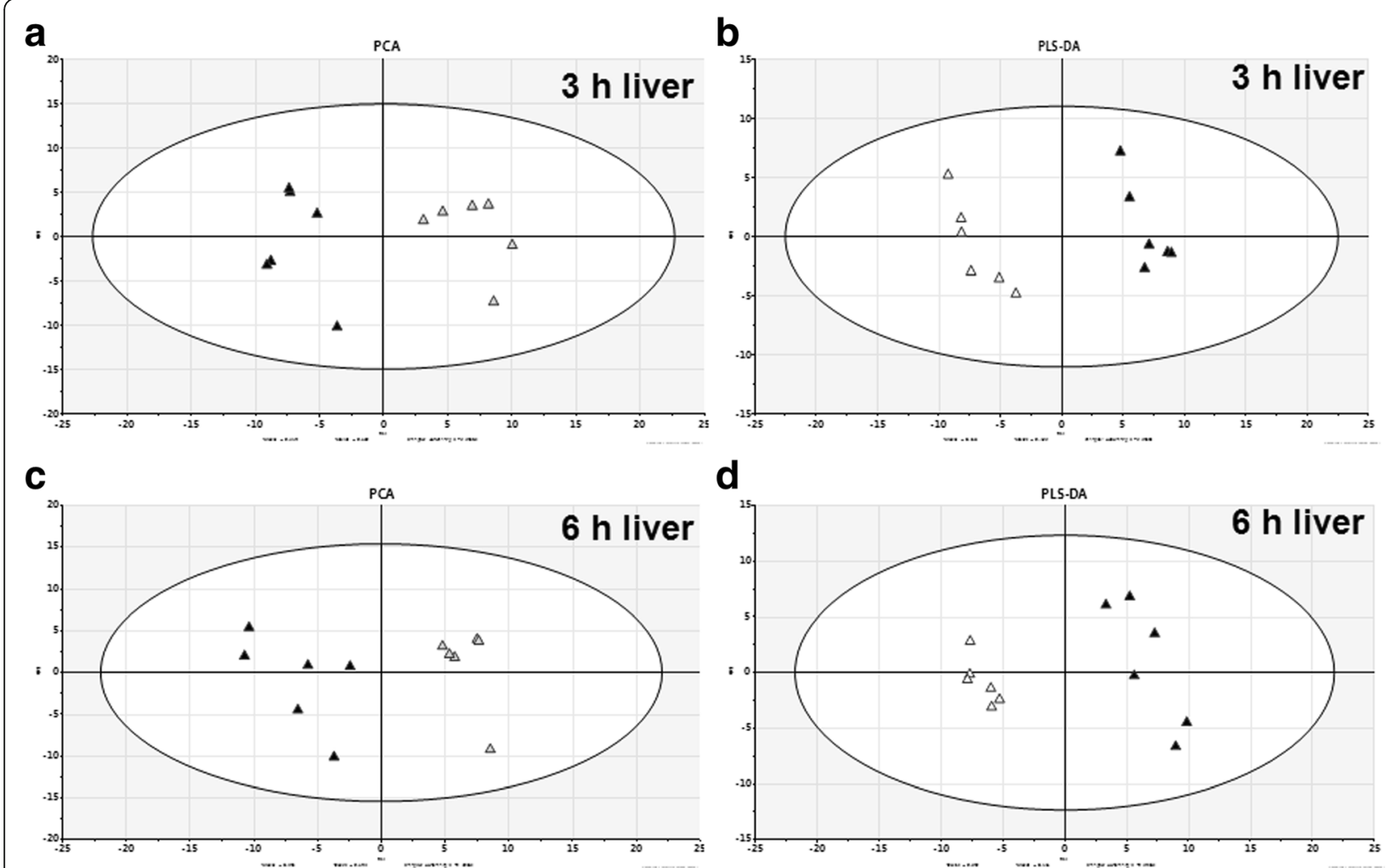

Fig. 5 PCA and PLS-DA score plot based on the liver PC and PE profiling of APAP-induced liver injured mice. One hundred six variables (63 PC/LPC and 43 PE/LPE) at $3 \mathrm{~h}(\mathbf{a}, \mathbf{c})$ and $6 \mathrm{~h}(\mathbf{b}, \mathbf{d})$ time points were loaded into a PCA $(\mathbf{a}, \mathbf{b})$ model and a PLS-DA (c, $\mathbf{d})$ model. The score plot indicated a clear separation between the saline- $(\triangle)$ and APAP-treated $(\boldsymbol{\Lambda})$ groups

by enzymatic and morphological evaluation. Consistently, the most dramatic change of $\mathrm{PC} / \mathrm{PE}$ profiles occurred at 3 $\mathrm{h}$ and $6 \mathrm{~h}$ time points, so varied PC/PE at both $3 \mathrm{~h}$ and $6 \mathrm{~h}$ time points were emphasized in our analysis.

As lipids serve many important functions, take part in several biochemical reactions and integrate diverse metabolic pathways, any alteration in lipids will reflect and affect cellular functions. Based on our results, the content of many $\mathrm{PC} / \mathrm{PE}$ species in plasma increased from $1 \mathrm{~h}$ time point, peaked at $3 \mathrm{~h}$ or $6 \mathrm{~h}$, and tended to return normality at $24 \mathrm{~h}$ time point. PC 33:1, PC 34:3, PE 34:2, PE 36:3, PE 38:4 and PE 38:6 were elevated significantly both at $3 \mathrm{~h}$ and $6 \mathrm{~h}$ time points upon APAP treatment. The increases of these 6 phospholipids were positively correlated with the changes of ALT/AST. The alternation of $\mathrm{PC} / \mathrm{PE}$ lipidome in plasma might be caused by damaging hepatocytes and/or interfering the lipid metabolism in liver. Based on our results, following an intoxication with APAP, many PC/PE species such as PC 33:1, PC 34:3, PE 34:2 and PE 36:3 in liver tissue decreased from $1 \mathrm{~h}$, appeared to be lowest at $6 \mathrm{~h}$, and had the tendency back to normal status at $24 \mathrm{~h}$ time point, which was opposite to their profile in plasma. Therefore, the increase of these phospholipids in plasma might be released by damaged hepatocytes in APAP-injured livers. However, some PEs increased in APAP-injured livers, which might cause the induction of certain PEs such as PE 38:4 and PE 38:6 in plasma.

The contents of PC/PE in liver rely on their degradation and synthesis. The degradation of $\mathrm{PC} / \mathrm{PE}$ results from the action of phospholipases. There are various phospholipases (PLA1, PLA2, PLC and PLD) that exhibit substrate specificities for different positions in phospholipids. Phospholipase A2 (PLA2) hydrolyzes the sn-2 ester bond in $\mathrm{PC} / \mathrm{PE}$, forming arachidonic acid and lysoPC/lyso-PE [17-19]. These bioactive lipid mediators play important roles in inflammation, phospholipid metabolism, and signal transduction, which participate in the progression of DILI. The PLA2 superfamily includes over twenty groups comprising such main types as the secreted sPLA2, cytosolic cPLA2, calcium-independent iPLA2, and so on. PC and PE also could be degraded by PLA1, PLC and PLD [20]. The decrease of PC contents might be the consequence of increased activities of phospholipases. Previous studies demonstrate that overdose of APAP may activate ${ } \mathrm{PLA}_{2}$ and $\mathrm{sPLA}_{2}$ [21-23], which are involved in APAP toxicity. The activated phospholipases degraded $\mathrm{PC}$ in livers. Our present study indicated that the mRNA levels 


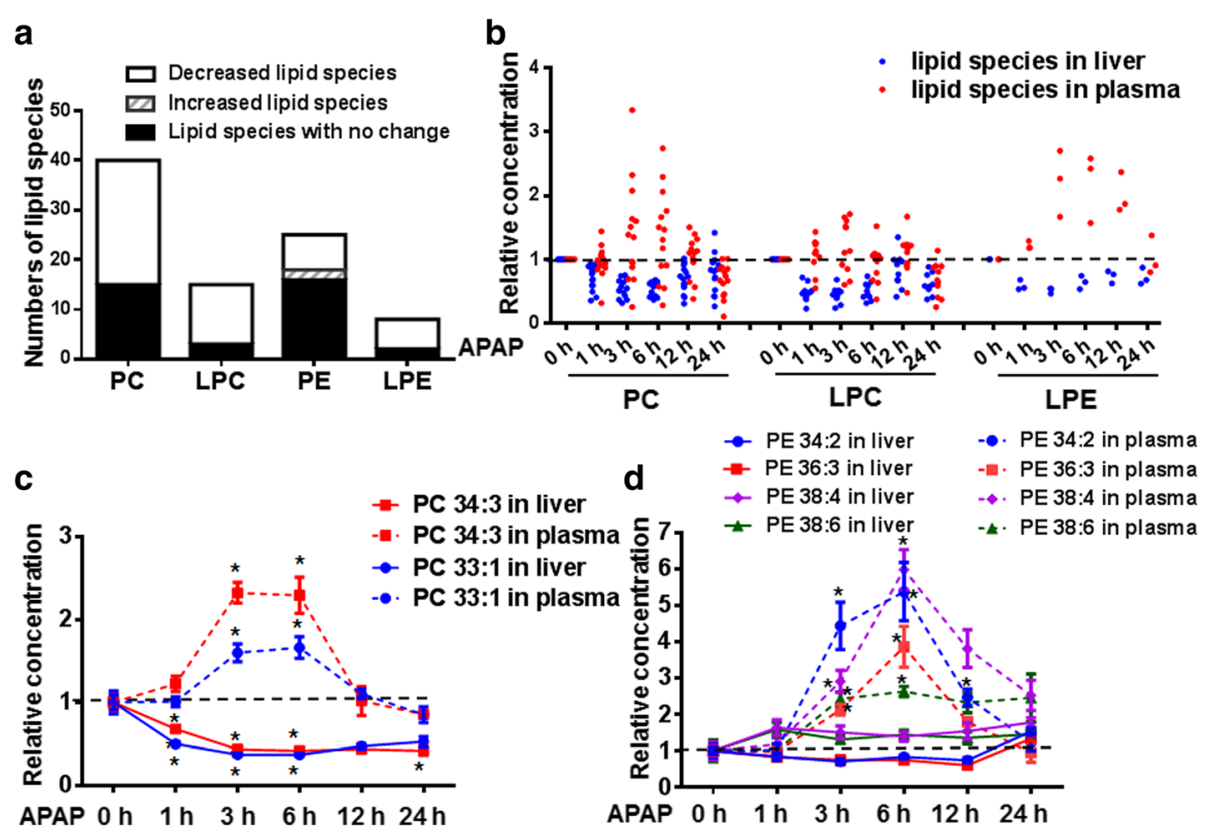

Fig. $\mathbf{}$ PC and PE profiles in the livers of APAP-treated mice. a The numbers of decreased and increased PCS/LPCS/PEs/LPEs in liver upon APAPtreatment were summarized. We defined lipid species with statistically significant decrease or increase at least at one time-point among $1 \mathrm{~h}, 3 \mathrm{~h}$ and $6 \mathrm{~h}$ as the decreased or increased lipid species respectively. $\mathbf{b}$ The decreased PCs/LPCs/PEs/LPEs in liver were picked out, and then Scatter Plot of these phospholipids both in liver and plasma were plotted. Because the significantly changed PEs in liver couldn't be detected in plasma, PEs were not shown in Fig. 6b. $\mathbf{c}$ and $\mathbf{d}$ The relative concentrations of 2 PCs (PC 33:1 and PC 34:3) and 4 PEs (PE 34:2, PE 36:3, PE 38:4 and PE 38:6) in liver of APAP induced liver-injured mice at $0 \mathrm{~h}, 1 \mathrm{~h}, 3 \mathrm{~h}, 6 \mathrm{~h}, 12 \mathrm{~h}$ and $24 \mathrm{~h}$ time points were presented. *statistically significant compared with saline-treated group

of phospholipases were suppressed by APAP treatment, which might be the adaptive protective reaction for the remaining alive hepatocytes.

Except for degradation, the contents of phospholipids in liver are also affected by their synthesis. The major pathway of PC synthesis is the CDP-choline pathway, also referred to as the "Kennedy pathway", which requires choline and three enzymes including choline kinase (Chka and Chkb), CTP:phosphocholine cytidylyltransferase (Pcyt) and diacylglycerol cholinephosphotransferase (Chpt). In liver, PC can also be generated endogenously in a second pathway via $\mathrm{PE}$ methylations catalyzed by hepatic phosphatidylethanolamine $\mathrm{N}$-methyltransferase (PEMT), which produces about one third of the PC in liver. PC also could be synthesized by reacylation of lyso-PC with lyso-PC acyltransferase (LPCAT) [24, 25]. We observed that the mRNA and protein levels of Chka, the rate-limiting enzyme of synthesizing $\mathrm{PC}$ de novo, were induced dramatically in APAP-injured mice. Chka was found to play a vital role in many biological signaling pathway, such as androgen receptor (AR) chaperone [26], and cell proliferation and carcinogenesis. Extensive studies of the structure and function of Chka show that the distal of the Chka promoter regions sequence is similar to the consensus of activated protein-1 binding site [27].In addition, APAP overdose causes nuclear accumulation of Hypoxia inducible factor-1 (HIF-1) in mouse livers as early as $1 \mathrm{~h}$ after treatment [28]. The expression of Chka was identified to be due to the regulation of transcriptional expression of HIF-1 [29]. HIF-1 deficiency mice were protected from APAP hepatotoxicity at $6 \mathrm{~h}$, but severe liver injury was observed at $24 \mathrm{~h}$, suggesting that HIF-1 is involved in the early stage of APAP toxicity [30]. Thus, the altered characteristic of Chka may be related to the alteration of associated transcriptional factors. The increased activities of phospholipases, decreased phospholipases mRNA expressions, and increased Chka mRNA/protein expression might partly explain why the contents of PC species in livers increased after APAP dosing and tended to recover at $24 \mathrm{~h}$ time point. PE is synthesized by four different pathways, the two quantitatively major of which are the CDP-ethanolamine pathway that PE catalyzed by ethanolamine kinase (ETNK1 and ETNK2) in the ER and the PS decarboxylation pathway that catalyzed by phosphatidylserine decarboxylase (PISD) in mitochondria [31]. The increase of some PE species in APAP-injured livers might be involved in the suppression of Pemt which is responsible one third of PC synthesis in liver. The dramatic decrease of Pemt may lead the accumulation of PE in APAP-injured liver.

Our study for the first time determined the dynamic $\mathrm{PC} / \mathrm{PE}$ changes both in plasma and liver that occurred 


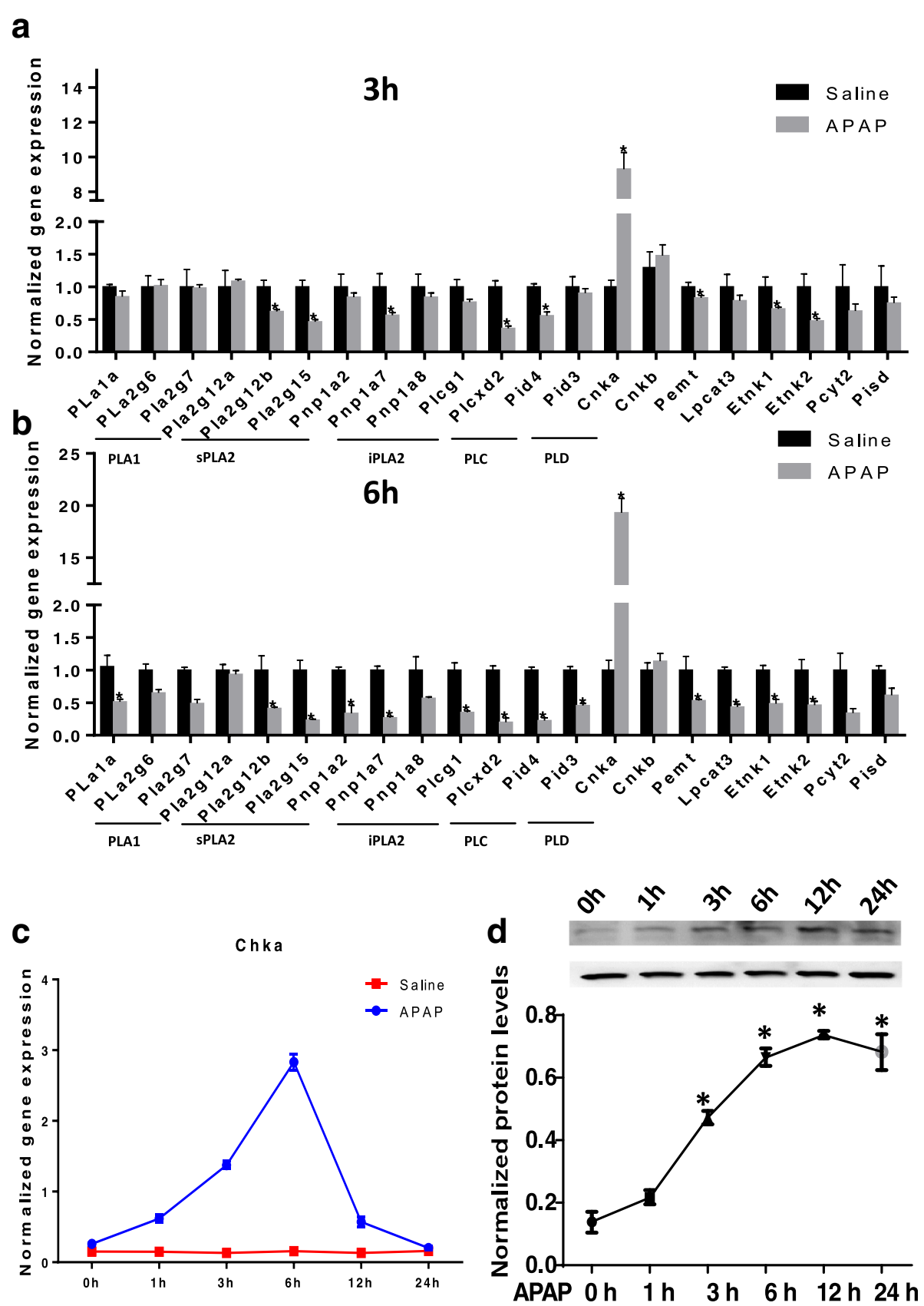

Fig. $\mathbf{7}$ The expression pattern of PC and PE metabolism related genes in APAP-injured mice liver (a). GRT-PCR analysis of the mRNA expressions of 13 phospholipases in livers treated by APAP for $3 \mathrm{~h}$ and $6 \mathrm{~h}$. $\mathbf{b}$ qRT-PCR analysis of the mRNA levels of PC/PE synthesis related genes in livers treated by APAP for $3 \mathrm{~h}$ and $6 \mathrm{~h}$. c qRT-PCR analysis of the mRNA level of Chka in livers treated by APAP for 0 h, 1 h, $3 \mathrm{~h}, 6 \mathrm{~h}, 12 \mathrm{~h}$ or $24 \mathrm{~h}$. $\mathbf{d}$ and e Western blot analysis of the protein levels of Chka in pooled liver samples treated by APAP for $0 \mathrm{~h}, 1 \mathrm{~h}, 3 \mathrm{~h}, 6 \mathrm{~h}, 12 \mathrm{~h}$ or $24 \mathrm{~h}$. $\mathrm{n}=6 \mathrm{per}$ time point. ${ }^{*} P<0.05$

in APAP-induced liver injury by lipidomics. This timecourse study provides a deeper understanding of the metabolic changes observed in APAP-induced liver injury. We took the plasma PC/PE lipidome, liver tissue $\mathrm{PC} / \mathrm{PE}$ lipidome, and $\mathrm{PC} / \mathrm{PE}$ metabolism-related gene expressions into consideration intergratedly. The comprehensive analysis might contribute to a better understanding the effect of APAP on lipid metabolism, hence finding potential therapeutic targets. In future studies, the concentration dependent changes of the PC/PE species in mice upon APAP treatment should be observed, which might provide valuable information for judging prognosis of DILI. Huo $\mathrm{T}$ et al. performed both UPLC-MS and (1)HNMRbased metabonomics analysis of serum samples from 
34 epileptic patients after valproate sodium treatment, and found that certain LPCs such as LPC 16:0, LPC 18:0, LPC 18:1 and LPC 18:2 decreased in valproate sodium induced liver injury [32]. The phospholipid profile of serum in human intoxicated with APAP also might be further observed by lipidomic techniques.

\section{Conclusions}

These results suggest that a novel targeted lipidomic method based on the metabolic profiling of phospholipid analyzed by LC-MS provides a better understanding the role of lipid metabolism in APAP-induced injured liver and serum, which might provide valuable information for judging prognosis of DILI and therapeutic targets.

\section{Methods}

\section{Animals}

C57BL/6 males aged 8-10 weeks and weighed $22-25 \mathrm{~g}$ at the time of the experimental procedures were purchased from the Shanghai Laboratory Animal Center, Chinese Academy Sciences (Shanghai, China). All mice were housed in microisolator cages under humidity$(50 \% \pm 5 \%)$ and temperature- $\left(24^{\circ} \pm 2{ }^{\circ} \mathrm{C}\right)$ controlled specific pathogen-free conditions with $12 \mathrm{~h}$ light/dark cycle. The mice were maintained on with free access to water and standard irradiated sterile chow.

\section{Treatment of mice}

Fresh acetaminophen (Sigma Aldrich, USA) solution was prepared for each experiment by dissolving acetaminophen in saline warmed to $59{ }^{\circ} \mathrm{C}$. Mice were fasted for $16 \mathrm{~h}$ and then injected intraperitoneally (i.p.) with saline or acetaminophen at $300 \mathrm{mg} / \mathrm{kg}$ (body weight) and food restored. APAP-treated mice were sacrificed and collected liver and plasma samples at the indicated time points $(0 \mathrm{~h}, 1 \mathrm{~h}, 3 \mathrm{~h}, 6 \mathrm{~h}, 12 \mathrm{~h}$, and $24 \mathrm{~h}, n=6 \sim 8$ each time point). Some mice were treated with saline as parallel controls at the same time points $(1 \mathrm{~h}, 3 \mathrm{~h}, 6 \mathrm{~h}, 12 \mathrm{~h}$, and $24 \mathrm{~h}, n=6 \sim 8$ each time point). Appropriate volume of PMSF stock solution in isopropanol $(100 \mathrm{mM})$ were added to freshly collected mouse plasma (5 $\mathrm{mM}$ in plasma) to stabilize the plasma lipidome. The livers were frozen in $-80{ }^{\circ} \mathrm{C}$ for later lipid/total RNA/protein extraction or fixed in $4 \%$ paraformaldehyde for tissue sections.

\section{Lipidomics analysis}

The lipids of plasma (50 uL) and liver samples $(200 \mathrm{mg})$ were extracted using liquid-liquid methyl tert-butyl ether (MTBE) extraction protocols developed by our group [15]. Briefly, endogenous free lipids including PE (17:0/ 17:0), PC (19:0/19:0), LPE 14:0, LPC 17:0 were selected as the internal standard for their corresponding lipid classes. The lipids of the mixture of the above mixed internal standard solution and samples were extracted twice using MTBE, dried and finally reconstituted in $200 \mu \mathrm{L}$ in the mixture of isopropanol/acetonitrile/ water $(75: 20: 5, v / v / v)$, then subjected to LC-MS analysis. An HPLC-quadrupole-time of flight hybrid mass system, which consisted of a LC-30 AD UFLC system (Shimadzu, Kyoto, Japan) and a TripleTOF 5600p quadrupole time of flight mass spectrometer (AB SCIEX, MA, USA), was used for lipidomic analysis. Chromatography was performed on a charged surface hybrid $\left(\mathrm{CSH}^{\mathrm{m}}\right) \mathrm{C} 18,2.1^{*} 100 \mathrm{~mm}, 1.7 \mathrm{um}$ UPLC column (Waters Corporation, USA) and subjected to a gradient elution as described previously. The eluents were monitored in positive electronic ion spray ESI mode. The lipid profiling was performed using the previous methods [33]. Briefly, the extracted ions chromatogram (EIC) of each lipid specie was created by applying the molecular weight (MW) lists (MW of quasi-molecular ions) generated from Lipidview ${ }^{\text {ti }}$ software (AB SCIEX, MA, USA). The peaks found in EIC were screened and identified according to the rules, including exact mass accuracy $(<5 \mathrm{ppm})$, specific fragment (PC and LPC have the specific product of 184.1; PE and LPE have the specific neutral loss of 141), retention time and isotope distribution pattern (similarity to the theoretical pattern). After corresponding IS correction, the areas of the identified peaks were used for quantitation.

\section{Biochemical assays and histopathology}

Alanine aminotransferase (ALT) and aspartate aminotransferase (AST) were measured by an automatic biochemical analyzer (SIEMENS ADVIA 1800: SIEMENS Healthcare Diagnostics, USA). Liver sections from saline- or APAP-treated mice were fixed in 4\% paraformaldehyde overnight and embedded in paraffin wax, sliced at $5 \mathrm{~mm}$ thickness and stained with hematoxylin and eosin (H\&E).

\section{Real-time RT-PCR (qRT-PCR) analysis}

Total RNA was prepared from mouse livers using Trizol reagent (Life Technologies, Thermo Fisher Scientific). Reverse transcription (RT) was performed using RevertAid ${ }^{\text {tw }}$ First Strand cDNA Synthesis Kit (Fermentas) according to the manufacturer's instructions. Relative expressions of indicated genes were determined by SYBR Green-based real-time PCR using Actb as an internal standard. A relative standard curve was used to calculate expression levels. Primers used for gene expression studies are listed in Table 1, and referenced in the primer bank [34].

\section{Western blot analysis}

Total protein was extracted from treated livers using radioimmune precipitation assay (RIPA) lysis buffer. The Chka protein level was detected by primary antibody 
Table 1 Primers sets used for qPCR

\begin{tabular}{|c|c|c|}
\hline Gene name & $\begin{array}{l}\text { Genbank } \\
\text { accession NM }\end{array}$ & Primer $\left(5^{\prime}-3^{\prime}\right)$ \\
\hline Actb & NM_007393.5 & $\begin{array}{l}\text { Forward: CGTGCGTGACATCAAAGAGAA } \\
\text { Reverse: GCTCGTTGCCAATAGTGATGA }\end{array}$ \\
\hline Pla1a & NM_134102.4 & $\begin{array}{l}\text { Forward: GGACTTTCTGTACTGCCCCT } \\
\text { Reverse: CGCAGGCTATTITCAGGTCC }\end{array}$ \\
\hline Pla2g12a & NM_001286948.1 & $\begin{array}{l}\text { Forward: GAAGCCTGTTCCACGCTATG } \\
\text { Reverse: CACTTGGTCAGGGAAGGGAT }\end{array}$ \\
\hline Pnpla2 & NM_001163689.1 & $\begin{array}{l}\text { Forward: ATGGTGCCCTATACTCTGCC } \\
\text { Reverse: TCTTCAGGGACATCAGGCAG }\end{array}$ \\
\hline Plcg1 & NM_021280.3 & $\begin{array}{l}\text { Forward: CCAGCAGAGAAACATGGCTC } \\
\text { Reverse: TCTTCCTCCTGAGCTGGTTG }\end{array}$ \\
\hline Pld3 & NM_001317355.1 & $\begin{array}{l}\text { Forward: TAAAGGTTCGCATCGCTGTG } \\
\text { Reverse: CTGATCCACCACCCAGAACT }\end{array}$ \\
\hline Pla2g12b & NM_023530.2 & $\begin{array}{l}\text { Forward: GGTGTCGATATGGAAAGGCG } \\
\text { Reverse: AACACTTGGTCATTGCTGGG }\end{array}$ \\
\hline Pnpla7 & NM_146251.4 & $\begin{array}{l}\text { Forward: ATCAAACAACGCCTGGGTTC } \\
\text { Reverse: GCCTGATACAGCACAATCCG }\end{array}$ \\
\hline Plcxd2 & NM_001134480.1 & $\begin{array}{l}\text { Forward: CTTCATCCACGGGCTCTTTG } \\
\text { Reverse: TCTTGAGGGTGCTGAGTGAG }\end{array}$ \\
\hline Pld4 & NM_178911.4 & $\begin{array}{l}\text { Forward: CAAGTGCCCATGAAACAGCT } \\
\text { Reverse: GCACCAAGTCCTTCACCTG }\end{array}$ \\
\hline Pla2g6 & NM_001199023.1 & $\begin{array}{l}\text { Forward: GTCCTGCTGCTCTGTAATGC } \\
\text { Reverse: TCCTTGCTGTGGATCTGGTT }\end{array}$ \\
\hline Pnpla8 & NM_026164.2 & $\begin{array}{l}\text { Forward: TGGTGGAGGAACAAGAGGTG } \\
\text { Reverse: TATGGCCCCTGTGCTTACTC }\end{array}$ \\
\hline Pla2g15 & NM_133792.2 & $\begin{array}{l}\text { Forward: GGCCTCCTGTTACCTCTGTT } \\
\text { Reverse: GGTTACCCAAATCACCAGGC }\end{array}$ \\
\hline Pla2g7 & NM_013737.5 & $\begin{array}{l}\text { Forward: CAGCTCAAGATCAAGGTCGC } \\
\text { Reverse: CAGCTTGCAGGAGTTGTCAG }\end{array}$ \\
\hline Pemt & NM_001290011.1 & $\begin{array}{l}\text { Forward: CGAGATGGGAGCAGAGAACT } \\
\text { Reverse: ATCTTGGGCTGGCTCATCAT }\end{array}$ \\
\hline Lpcat3 & NM_145130.2 & $\begin{array}{l}\text { Forward: CCAGGGAAGATGCCAAACAG } \\
\text { Reverse: TAGTCGTCTGTGATGTGGGG }\end{array}$ \\
\hline Chka & NM_001271496.1 & $\begin{array}{l}\text { Forward: CTTCGCGAGGACCAGTTC } \\
\text { Reverse: CTATGGAGTCTGGCAGGGAG }\end{array}$ \\
\hline Pcyt2 & NM_024229.2 & $\begin{array}{l}\text { Forward: TGGTGCGATGGCTGCTATG } \\
\text { Reverse: CCCTTATGCTTGGCAATCTCC }\end{array}$ \\
\hline Pisd & NM_177298.3 & $\begin{array}{l}\text { Forward: CATACTGCTCCTGTCCGATCC } \\
\text { Reverse: TTCCGTTCCCTGTACTTCTCATA }\end{array}$ \\
\hline Etnk1 & NM_029250.2 & $\begin{array}{l}\text { Forward: TGAGGATTTACGGCAACAAGAC } \\
\text { Reverse: CAGTCCGTATTAAAGGTGCAGT }\end{array}$ \\
\hline Etnk2 & NM_175443.5 & $\begin{array}{l}\text { Forward: CGGTGGAACAGGACGACATC } \\
\text { Reverse: AGGCCAATAGCTTGTTGGTGA }\end{array}$ \\
\hline Chkb & NM_007692.6 & $\begin{array}{l}\text { Forward: GCCGAGCCTATCAGTGGTG } \\
\text { Reverse: GTAGTGAGCATCGGAAGAGCA }\end{array}$ \\
\hline
\end{tabular}

against Chka (Proteintech Group, Inc. Rosemont, IL, USA) using Western blot assay according to published methods [35]. $\beta$-actin was used as loading control.

\section{Data analysis}

MultiQuant ${ }^{\mathrm{TM}}$ software (Version 2.1.1, AB SCIEX, MA, USA), calculated the peak area ratios of lipid species and their corresponding internal standards, as the concentrations of lipid species. PCA and PLS-DA were performed using SIMCA-P 12.0 software (Umetrics, Umea, Sweden). The original data of PC/PE concentrations and the fold changes of APAP-treated mice compared with saline-treated mice at indicated time points in plasma and liver were shown in Supplemental tables (Additional file 1: Table S1 and Additional file 4: Table S2) respectively. The original data were presented as the mean $\pm \mathrm{SD}$. To compare the concentrations of $\mathrm{PC} / \mathrm{PE}$ between the APAP-treated group and saline-treated group in each indicated time point, statistical analysis was performed with t-tests for unpaired data, the statistical significance was corrected for multiple comparisons using the Holm-sidak method by using GraphPad Prism 6.0.

\section{Additional files}

Additional file 1: Table S1. The original data of PC/PE concentrations and the fold changes of APAP-treated mice compared with saline-treated mice at indicated time points in plasma. The original data were presented as the mean \pm SD. The lipid species with statistical significance were labeled with red. (DOCX $32 \mathrm{~kb}$ )

Additional file 2: Figure S1. Heat map for $P C$ and $P E$ profiles in the plasma of APAP-treated mice. The mean of the concentration of PC(A)/ $\mathrm{PE}(\mathrm{B})$ in plasma of APAP-treated mice at indicated time point $(n=6 \sim 8$ for each time point) were normalized to the saline-treated group at the same time point. The color of each section is proportional to the relative concentration of phospholipids as shown in the color bar (blue-black-yellow colors correspond to low-moderate-high levels respectively). Rows: phospholipid species; columns: samples at indicated time point. (TIFF $48 \mathrm{~kb}$ )

Additional file 3: Figure S2. Heat map for $P C$ and $P E$ profiles in the livers of APAP-treated mice. The mean of the concentration of PC(A)/ $\mathrm{PE}(\mathrm{B})$ in livers of APAP-treated mice at indicated time point $(n=6 \sim 8$ for each time point) were normalized to the saline-treated group at the same time point. The color of each section is proportional to the relative concentration of phospholipids as shown in the color bar (blue-black-yellow colors correspond to low-moderate-high levels respectively). Rows: phospholipid species; columns: samples at indicated time point. (TIFF $57 \mathrm{~kb}$ )

Additional file 4: Table S2. The original data of PC/PE concentrations and the fold changes of APAP-treated mice compared with saline-treated mice at indicated time points in liver. The original data were presented as the mean \pm SD. The lipid species with statistical significance were labeled with red. (DOCX $39 \mathrm{~kb})$

\section{Acknowledgements}

None

\section{Funding}

This work was supported by the Major Project of National Twelfth Five Plan (2012ZX09303-001 and 2013ZX10002004), National Natural Science Foundation of China (NSFC 81270497).

\section{Availability of data and materials}

Not applicable.

\section{Authors' contributions}

YM M and XB L designed and wrote the paper. YN M performed the majority of research. All authors have read and approved the final manuscript.

Ethics approval and consent to participate

All experiments were performed with the approval of Shanghai Jiaotong University School of medicine animal ethics committee. 


\section{Consent for publication}

Not applicable.

\section{Competing interests}

The authors declare that they have no competing interests.

\section{Publisher's Note}

Springer Nature remains neutral with regard to jurisdictional claims in published maps and institutional affiliations.

\section{Author details}

'Division of Gastroenterology and Hepatology, Renji Hospital, School of Medicine, Shanghai Jiao Tong University, Shanghai Institute of Digestive Disease, Shanghai, China. 'Department of Pharmacology, School of Medicine, Shanghai Jiao Tong University, Institute of Medical Sciences, Shanghai, China. ${ }^{3}$ Department of Interventional Oncology, Renji Hospital, School of Medicine, Shanghai Jiao Tong University, Shanghai, China. ${ }^{4}$ Department of Physiology and Pathophysiology, School of Basic Medical Sciences, Fudan University, Shanghai 200032, China. ${ }^{5}$ Division of Gastroenterology and Hepatology, Xinhua Hospital, School of Medicine, Shanghai Jiao Tong University, Shanghai, China.

\section{Received: 23 February 2017 Accepted: 2 August 2017}

\section{Published online: 14 August 2017}

\section{References}

1. Lancaster EM, Hiatt JR, Zarrinpar A. Acetaminophen hepatotoxicity: an updated review. Arch Toxicol. 2015:89:193-9.

2. Stephens C, Andrade RJ, Lucena MI. Mechanisms of drug-induced liver injury. Curr Opin Allergy Clin Immunol. 2014;14:286-92.

3. Yuan L, Kaplowitz N. Mechanisms of drug-induced liver injury. Clin Liver Dis. 2013;17:507-18. vii

4. Begriche K, Massart J, Robin MA, Borgne-Sanchez A, Fromenty B. Drug-induced toxicity on mitochondria and lipid metabolism: mechanistic diversity and deleterious consequences for the liver. J Hepatol. 2011:54:773-94.

5. Shimizu Y. Effect of carbon tetrachloride administration on the synthesis of triglycerides and phospholipids in rat liver. J Lipid Res. 1969:10:479-86.

6. Sugano M, Cho S, Imaizumi K, Wada M. Hepatotoxicity and lipid metabolism. 3. Changes in phosphatidylcholine and phosphatidylethanolamine during hepatic injury caused by carbon tetrachloride. Biochem Pharmacol. 1970;19:2325-33.

7. Griffiths WJ, Ogundare M, Williams CM, Wang Y. On the future of "omics": lipidomics. J Inherit Metab Dis. 2011;34:583-92.

8. Han X. Lipidomics: developments and applications. J Chromatogr B Analyt Technol Biomed Life Sci. 2009;877:2663.

9. Rolim AE, Henrique-Araujo R. Ferraz EG, de Araujo Alves Dultra FK Fernandez LG: Lipidomics in the study of lipid metabolism: current perspectives in the omic sciences. Gene. 2015:554:131-9.

10. Sun J, Slavov S, Schnackenberg LK, Ando Y, Greenhaw J, Yang X, Salminen W, Mendrick DL, Beger R. Identification of a metabolic biomarker panel in rats for prediction of acute and idiosyncratic hepatotoxicity. Comput Struct Biotechnol J. 2014;10:78-89.

11. Peng J, Cai S, Wang L, Zhao N, Zhang TJ, Chen ZX, Meng FH. A metabonomic analysis of serum from rats treated with ricinine using ultra performance liquid chromatography coupled with mass spectrometry. PLoS One. 2014;9:e90416.

12. Gonzalez E, van Liempd S, Conde-Vancells J, Gutierrez-de Juan V, PerezCormenzana M, Mayo R, Berisa A, Alonso C, Marquez CA, Barr J, et al. Serum UPLC-MS/MS metabolic profiling in an experimental model for acute-liver injury reveals potential biomarkers for hepatotoxicity. Metabolomics. 2012;8: 997-1011.

13. Cheng J, Ma X, Krausz KW, Idle JR, Gonzalez FJ. Rifampicin-activated human pregnane $X$ receptor and CYP3A4 induction enhance acetaminopheninduced toxicity. Drug Metab Dispos. 2009;37:1611-21.

14. Xie T, Zhou X, Wang S, Lu Y, Zhu H, Kang A, Deng H, Xu J, Shen C, Di L, Shan J. Development and application of a comprehensive lipidomic analysis to investigate Tripterygium wilfordii-induced liver injury. Anal Bioanal Chem. 2016:408:4341-55.

15. Wang X, Gu X, Song H, Song Q, Gao X, Lu Y, Chen H. Phenylmethanesulfonyl fluoride pretreatment stabilizes plasma lipidome in lipidomic and metabolomic analysis. Anal Chim Acta. 2015;893:77-83.
16. Jaeschke $H$, Xie Y, McGill MR. Acetaminophen-induced liver injury: from animal models to humans. J Clin Transl Hepatol. 2014:2:153-61.

17. Caro AA, Cederbaum Al. Role of cytochrome P450 in phospholipase A2and arachidonic acid-mediated cytotoxicity. Free Radic Biol Med. 2006:40: 364-75.

18. Ishihara K, Kanai S, Tanaka K, Kawashita E, Akiba S. Group IVA phospholipase A2 deficiency prevents CCl4-induced hepatic cell death through the enhancement of autophagy. Biochem Biophys Res Commun. 2016;471:15-20.

19. Moon TC, Hwang HS, Quan Z, Son KH, Kim CH, Kim HP, Kang SS, Son JK, Chang HW. Ochnaflavone, naturally occurring biflavonoid, inhibits phospholipase A2 dependent phosphatidylethanolamine degradation in a CCl4-induced rat liver microsome. Biol Pharm Bull. 2006;29:2359-61.

20. Kim NY, Kim TH, Lee E, Patra N, Lee J, Shin MO, Kwack SJ, Park KL, Han SY, Kang TS, et al. Functional role of phospholipase D (PLD) in di(2-ethylhexyl) phthalate-induced hepatotoxicity in Sprague-Dawley rats. J Toxicol Environ Health A. 2010;73:1560-9.

21. Reilly TP, Bourdi M, Brady JN, Pise-Masison CA, Radonovich MF, George JW, Pohl LR. Expression profiling of acetaminophen liver toxicity in mice using microarray technology. Biochem Biophys Res Commun. 2001;282:321-8.

22. Bhave VS, Donthamsetty S, Latendresse JR, Cunningham ML, Mehendale HM. Secretory phospholipase a(2)-mediated progression of hepatotoxicity initiated by acetaminophen is exacerbated in the absence of hepatic COX-2. Toxicol Appl Pharmacol. 2011;251:173-80

23. Chaudhuri S, McCullough SS, Hennings L, Brown AT, Li SH, Simpson PM, Hinson JA, James LP. Effect of trifluoperazine on toxicity, HIF-1alpha induction and hepatocyte regeneration in acetaminophen toxicity in mice. Toxicol Appl Pharmacol. 2012;264:192-201.

24. Li Z, Agellon LB, Vance DE. Phosphatidylcholine homeostasis and liver failure. J Biol Chem. 2005:280:37798-802.

25. Cole LK, Vance JE, Vance DE. Phosphatidylcholine biosynthesis and lipoprotein metabolism. Biochim Biophys Acta. 2012;1821:754-61.

26. Asim M, Massie CE, Orafidiya F, Pertega-Gomes N, Warren AY, Esmaeili M, Selth LA, Zecchini HI, Luko K, Qureshi A, et al. Choline Kinase alpha as an androgen receptor chaperone and prostate cancer therapeutic target. J Nat Cancer Inst. 2016;108

27. Aoyama C, Liao H, Ishidate K. Structure and function of choline kinase isoforms in mammalian cells. Prog Lipid Res. 2004:43:266-81.

28. James LP, Donahower B, Burke AS, McCullough S, Hinson JA. Induction of the nuclear factor HIF-1alpha in acetaminophen toxicity: evidence for oxidative stress. Biochem Biophys Res Commun. 2006;343:171-6.

29. Glunde K, Shah T, Winnard PT Jr, Raman V, Takagi T, Vesuna F, Artemov D, Bhujwalla ZM. Hypoxia regulates choline kinase expression through hypoxia-inducible factor-1 alpha signaling in a human prostate cancer model. Cancer Res. 2008:68:172-80.

30. Sparkenbaugh EM, Saini Y, Greenwood KK, LaPres JJ, Luyendyk JP, Copple BL, Maddox JF, Ganey PE, Roth RA. The role of hypoxia-inducible factor1alpha in acetaminophen hepatotoxicity. J Pharmacol Exp Ther. 2011;338: 492-502

31. Vance JE, Tasseva G. Formation and function of phosphatidylserine and phosphatidylethanolamine in mammalian cells. Biochim Biophys Acta. 2013; 1831:543-54.

32. Huo T, Chen X, Lu X, Qu L, Liu Y, Cai S. An effective assessment of valproate sodium-induced hepatotoxicity with UPLC-MS and (1)HNMR-based metabonomics approach. J Chromatogr B Analyt Technol Biomed Life Sci. 2014:969:109-16.

33. Chen S, Hoene M, Li J, Li Y, Zhao X, Haring HU, Schleicher ED, Weigert C, Xu $\mathrm{G}$, Lehmann R. Simultaneous extraction of metabolome and lipidome with methyl tert-butyl ether from a single small tissue sample for ultra-high performance liquid chromatography/mass spectrometry. J Chromatogr A. 2013:1298:9-16

34. Spandidos A, Wang $X$, Wang $H$, Seed B. PrimerBank: a resource of human and mouse PCR primer pairs for gene expression detection and quantification. Nucleic Acids Res. 2010;38:D792-9.

35. Wang $Z$, Yang $X$, Chen L, Zhi $X$, Lu H, Ning Y, Yeong J, Chen S, Yin L, Wang $X, L i X$. Upregulation of hydroxysteroid sulfotransferase $2 \mathrm{~B} 1 \mathrm{~b}$ promotes hepatic oval cell proliferation by modulating oxysterol-induced LXR activation in a mouse model of liver injury. Arch Toxicol. 2017;91(1):271-87. 